\title{
Éléphantiasis pénoscrotal idiopathique : une nouvelle observation et revue de la littérature
}

\author{
Angoran Dekou, MD ; Paul-Gérard Konan, MD ; Blaise Yao, MD ; Clément Vodi, MD ; \\ Abroulaye Fofana, MD; Konan Manzan, MD
}

Département de néphro-urologie, université de Cocody, Abidjan, Côte d'Ivoire

Cite as: Can Urol Assoc J 2013;7(1):E29-E32. http://dx.doi.org/10.5489/cuaj.11168

\section{Résumé}

Nous rapportons un nouveau cas d'éléphantiasis pénoscrotal idiopathique chez un adulte de 31 ans. La masse scrotale atteignait les genoux, avec lésions de grattage traitées et guéries à l'éosine aqueuse associée à une antibiothérapie par voie parentérale. Une exérèse de la masse scrotale a été effectuée, suivie d'une plastie de peau scrotale postérosupérieure associée à une circoncision partielle. Le résultat esthétique et fonctionnel à long terme fut excellent, avec une peau pénienne assouplie et élastique.

\section{Introduction}

L'éléphantiasis des organes génitaux externes est caractérisé par une lymphangite chronique avec hypertrophie spectaculaire des organes génitaux externes. C'est une affection rare d'étiologie variée dont la plus fréquente est la filariose. L'étiologie en dehors des zones d'endémie filarienne est toujours difficile à retrouver. II s'agit d'une affection qui a un retentissement sur les activités sexuelles, professionnelles et sportives, et pose un problème esthétique $\mathrm{e}^{1-10}$.

Ainsi, la découverte $d^{\prime}$ un éléphantiasis qui évolue depuis plusieurs années pose particulièrement le problème du traitement chirurgical ${ }^{1-10}$. Nous rapportons un nouveau cas d'éléphantiasis pénoscrotal idiopathique dont nous présentons, à travers une revue de la littérature, l'épidémiologie, la présentation clinique, le traitement et le résultat à long terme.

\section{Observation}

NJ, âgé de 31 ans, vivant en zone rurale, avait consulté pour grosses bourses chroniques, évoluant depuis l'âge de 18 ans. L'anamnèse a révélé qu'il s'agissait d'une lésion $\mathrm{d}^{\prime}$ installation progressive avec pour point de départ du prurit scrotal suivi de lésions de grattage, d'une augmentation progressive et indolore du volume des bourses avec épaississement du scrotum étendu au pénis dans un contexte apyrétique et traité à l'indigénat. Handicapé par cette énorme masse, le patient a suspendu toute activité, notamment les activités scolaires et sportives. Un homme de bonne volonté l'ayant découvert l'a accompagné et fait hospitaliser au Service d'urologie du CHU de Cocody d'Abidjan, le 6 janvier 2008. L'examen clinique a révélé un patient avec un état de santé général moyen, une température à $36,8{ }^{\circ} \mathrm{C}$, une TA de 120/80 mm hg et une hypertrophie pénoscrotale avec pachydermie. Le diagnostic d'éléphantiasis a été retenu. On a noté par ailleurs des lésions scrotales de grattage surinfectées (fig.1) traitées avec un antibiotique par voie parentérale (céphalosporine de $3^{\mathrm{e}}$ génération) pendant 5 jours, associé à de l'éosine aqueuse en traitement local après désinfection de la plaie, tous les deux ou trois jours. La guérison de la plaie cutanée a été obtenue au bout de deux semaines, avec une masse scrotale modérément diminuée de volume avec cependant une peau périnéale et scrotale postérieure souple (fig. 2). La recherche de microfilaires dans le sang a donné un résultat négatif et l'échographie abdominopelvienne était normale. Cependant, I'échographie scrotale a montré des testicules apparemment normaux en situation normale mais invaginés dans une épaisseur occupant entièrement les bourses. L'étiologie n'a pu être retrouvée. Le bilan préopératoire était apparemment normal. Le patient a été opéré le 28 janvier 2008 sous rachianesthésie. L'opération a consisté en une incision scrotale transversale 2 à $3 \mathrm{~cm}$ en dessous du sillon pénoscrotal, puis remontant latéralement vers les orifices externes des canaux inguinaux. La dissection du plan profond était progressive avec une hémostase progressive aux fils Vicryl $n^{\circ} 2 / 0$ ou 3/0, parfois au bistouri électrique, des vaisseaux très dilatés de la peau scrotale et de l'épaisseur invaginée. L'exérèse de la masse s'est faite avec conservation de la paroi postérosupérieure du scrotum qui a été rabattue en avant, couvrant les testicules apparemment normaux, et suturée à la base du pénis, sur drain aspiratif, après contrôle de I'hémostase et orchidopexie bilatérale. Une ablation par- 


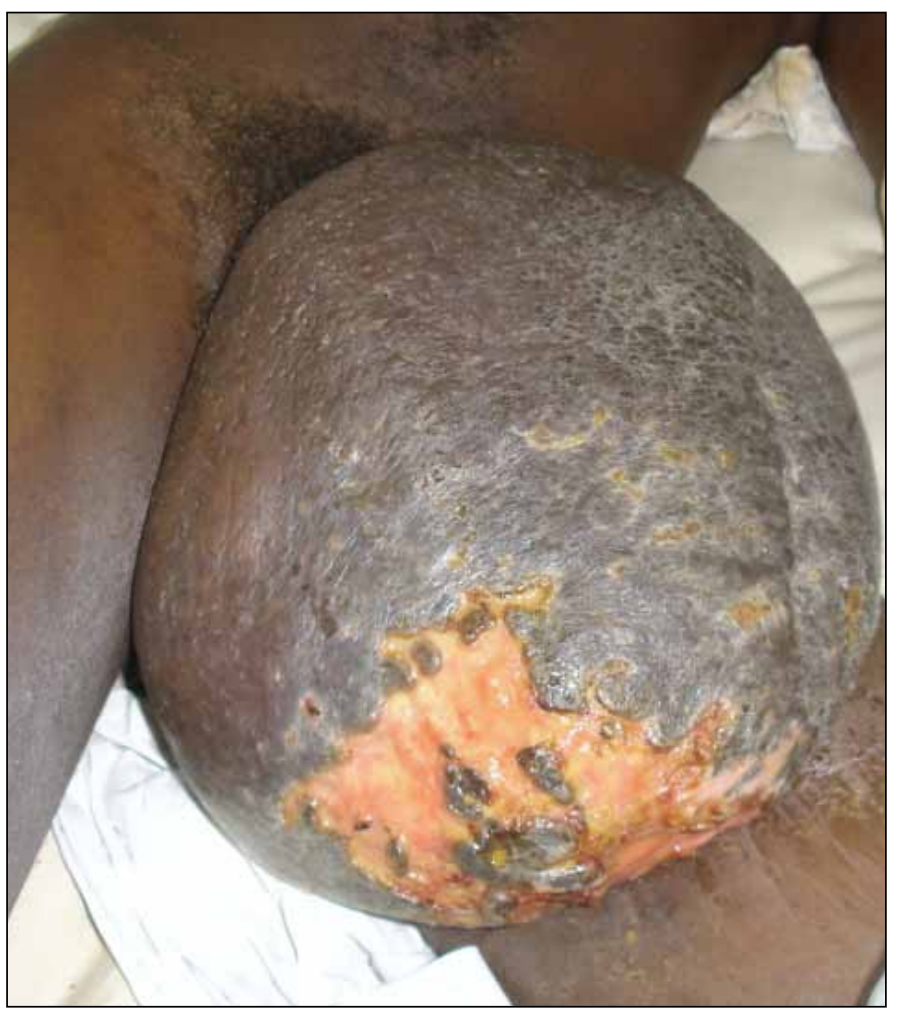

Fig. 1 : Aspect clinique de l'éléphantiasis pénoscrotal avec lésions de grattage.

tielle du prépuce a également été réalisée, libérant le méat urétral. Le drain a été enlevé au $3^{\mathrm{e}}$ jour postopératoire. Au $8^{\mathrm{e}}$ jour postopératoire, il s'est produit un lâchage pariétal qui a été avivé (fig. 3) et suturé sous anesthésie locale. La cicatrisation a été effective avec ablation totale des fils au $11^{\mathrm{e}}$ jour de la reprise du lâchage pariétal (fig. 4). Avec un recul de 3 ans et 10 mois, soit le 11 novembre 2011, les résultats esthétiques et fonctionnels sont bons : la peau pénoscrotale est devenue souple et normale (fig. 5). Le patient note des rapports sexuels satisfaisants malgré la déformation du gland, et signale avoir une fillette de 19 mois.

\section{Commentaire}

Nous rapportons un cas d'éléphantiasis chez un adulte de 31 ans, sans cause retrouvée, et qui a été efficacement traité par exérèse chirurgicale et plastie scrotale de recouvrement.

En effet, l'éléphantiasis des OGE est une affection rare, mais plus fréquente dans les zones tropicales, d'endémie filarienne. Il est la conséquence d'une obstruction mécanique des canaux lymphatiques, soit par la présence de vers adultes de type filaire, par inflammation et fibrose des vaisseaux lymphatiques ou par une compression externe $e^{1-4}$. Il atteint particulièrement les adultes et touche préférentiellement l'homme ${ }^{5-8}$. Hormis les causes parasitaires, l'éléphantiasis des OGE peut être de cause soit bactérienne ou congénitale, soit iatrogène ou idiopathique ${ }^{2-7}$, enfin soit

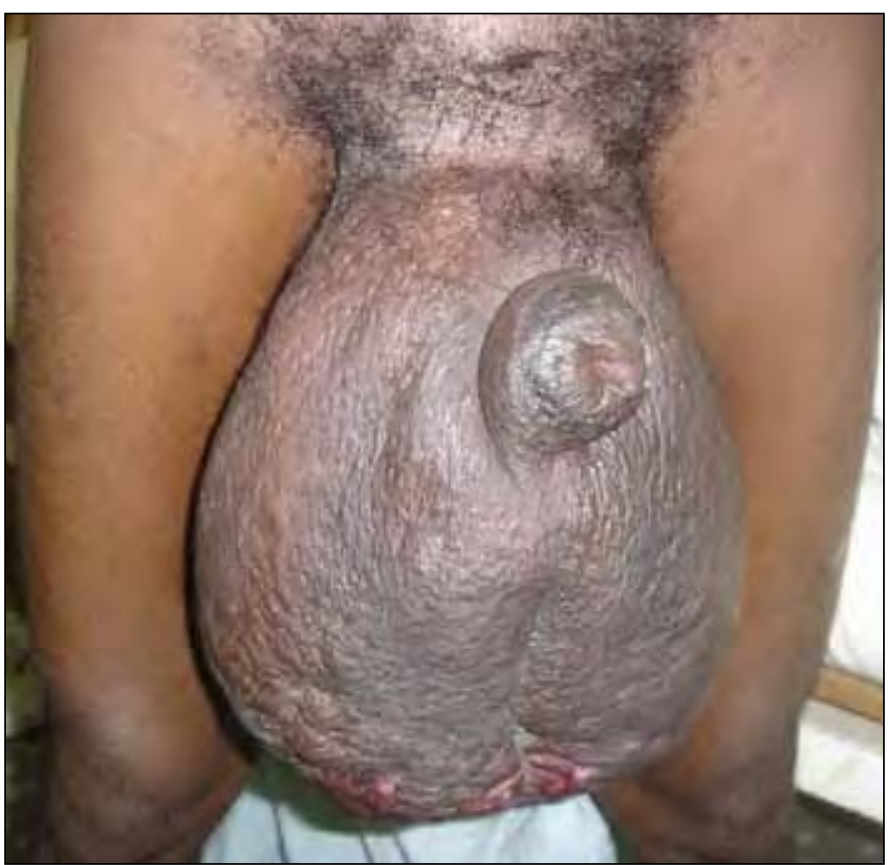

Fig. 2 : Éléphantiasis des OGE, après guérison des lésions de grattage.

secondaire à une sténose inflammatoire de l'urètre, cause la plus fréquente rapportée dans l'étude de Ndoye et al. ${ }^{6}$. L'étiologie chez notre patient n'a pu être dégagée.

Sur le plan clinique, l'éléphantiasis rapporté dans la littérature $^{1-10}$ est lié à une hypertrophie parfois monstrueuse du scrotum souvent accompagné d'un enfouissement du pénis.

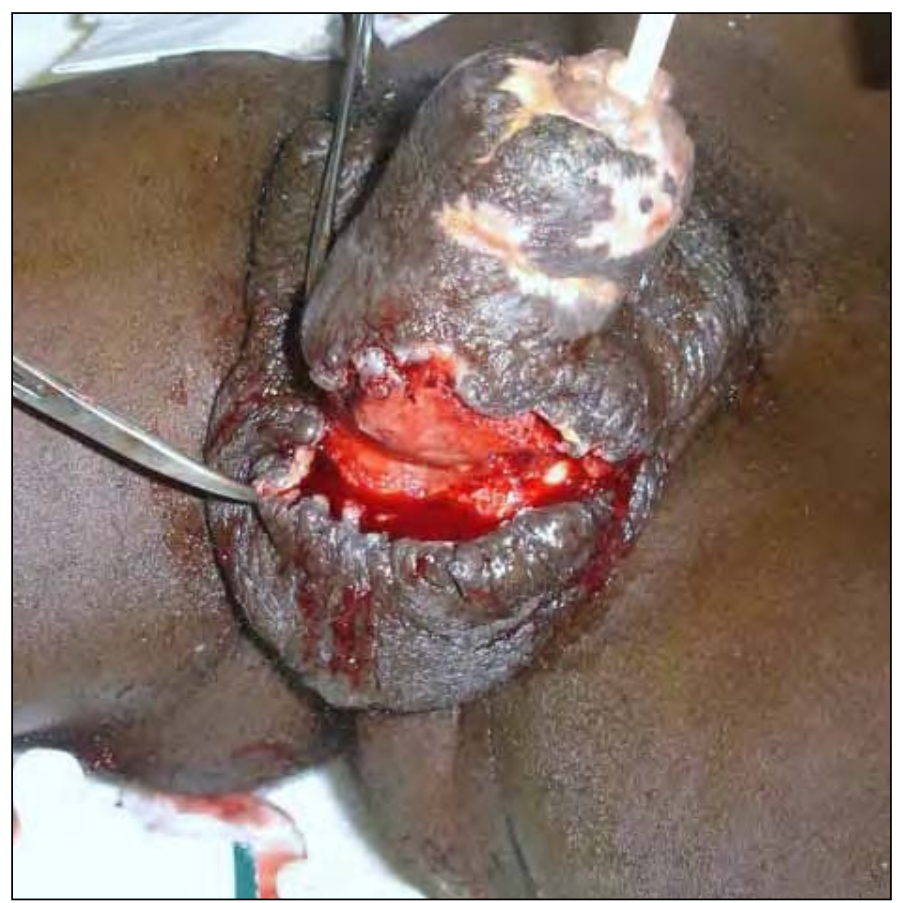

Fig. 3 : Lâchage de suture pariétale, avivement des berges cutanées sous anesthésie locale (J8 postopératoire). 


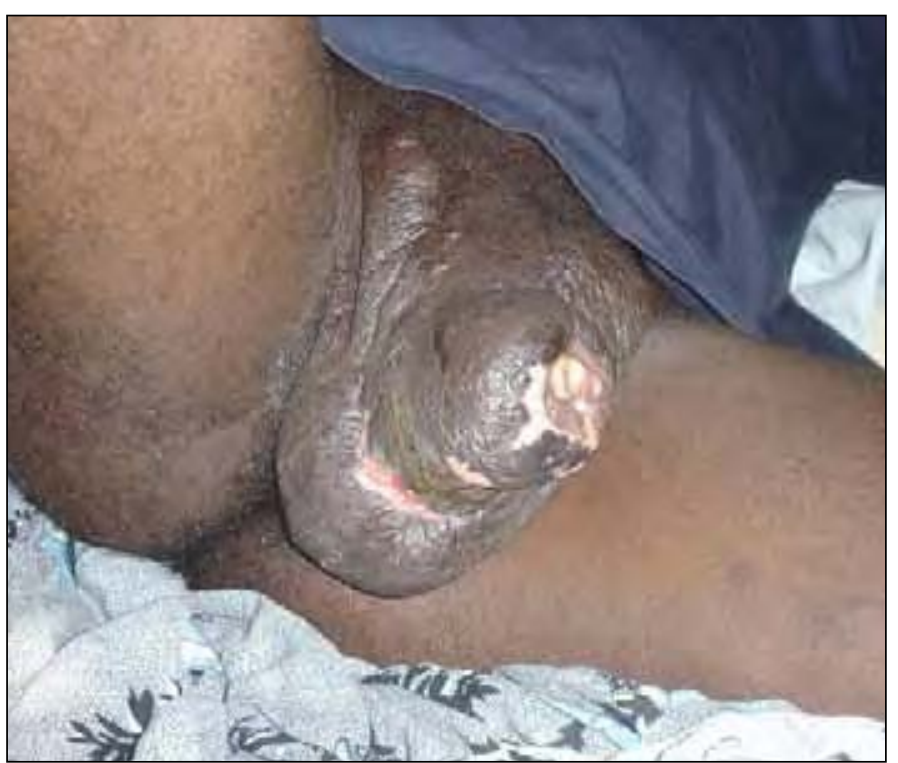

Fig. 4 : Cicatrisation de la plaie opératoire (J19 postopératoire).

Cette hypertrophie des organes génitaux externes associe une pachydermie qui se caractérise par un épaississement de la paroi scrotale qui est cartonnée, épargnant le plus souvent la peau scrotale postérosupérieure comme le rapportent tous les auteurs, et qui sert de tissu de recouvrement en cas d'exérèse suivie de plastie. Le délai de consultation est souvent long, soit deux à quinze ans selon les auteurs ${ }^{3,6}$; il est de 8 ans chez notre patient qui présentait les caractéristiques cliniques décrites dans la littérature ${ }^{1-10}$.

La découverte d'un éléphantiasis des organes génitaux externes pose un problème esthétique et constitue un handicap physique et fonctionnel qui impose la reconstruction plastique. L'étiologie est le plus souvent difficile à retrouver, en particulier en dehors des zones d'endémie filarienne ${ }^{4-8}$. La recherche étiologique implique selon les circonstances la réalisation d'examens complémentaires parfois onéreux pour nos patients pauvres qui prennent en charge leurs soins de santé. Ces examens sont pourtant utiles pour la recherche étiologique. Ce sont comme le rapporte la litté-

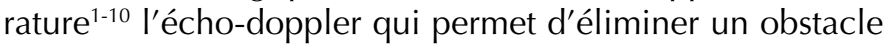
sur l'axe vasculaire des membres inférieurs, en particulier en cas de filariose, la recherche de filaires adultes et la recherche sanguine de microfilaires. La lymphographie bipédieuse est de moins en moins utilisée. L'échographie, la tomodensitométrie abdominale et même l'imagerie par résonance magnétique et nucléaire (IRMN) permettent d'éliminer une cause compressive telle une tumeur pelvienne ou abdominale. L'urétrocystographie rétrograde et mictionnelle (UCRM) est demandée lorsqu'on soupçonne une sténose scléro-inflammatoire de l'urètre. Chez notre patient, nous avons fait une recherche de microfilaires dans le sang et réalisé une échographie abdominopelvienne qui ont donné des résultats normaux. Même si l'étiologie est retrouvée,

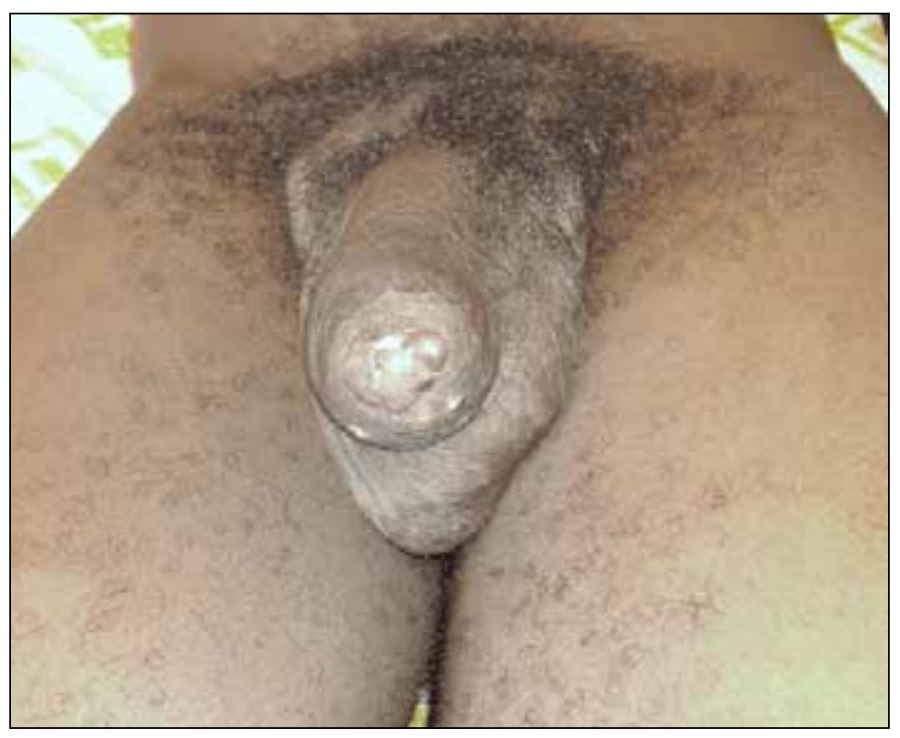

Fig. 5 : Aspect pénoscrotal près de 4 ans plus tard.

I'hypertrophie doit être si possible traitée ${ }^{4}$. Mais comme le rapporte Zugor et al. ${ }^{4}$, le but du traitement de l'éléphantiasis est de réparer l'obstruction en rétablissant la perméabilité lymphatique, puis de corriger la dysmorphie. Aucun traitement médicamenteux n'est efficace. Seule la chirurgie est recommandée.

Le traitement chirurgical proposé selon les auteurs ${ }^{1-10}$ consiste à exciser la masse, en emportant toute la peau et les tissus sous-cutanés pathologiques. La reconstruction de la peau de la verge se fait par des greffes cutanées et celle de la bourse scrotale par des greffes ou des lambeaux locaux de peau.

Les techniques opératoires sont nombreuses : certaines sont abandonnées telles les techniques conservatrices visant à améliorer le drainage lymphatique, notamment la lymphangioplastie, ou l'anastomose lymphatico-veineuse (opération de Nielubowicz) entre la crosse de la veine saphène interne et le groupe lymphonodal inguinal superficiel, dont la perméabilité de l'anastomose est temporaire, ainsi que la greffe d'épiploon ${ }^{1,10}$. D'autres, telles l'exérèse chirurgicale et la plastie de recouvrement, restent de mise. De nombreuses techniques ont été décrites. Elles consistent en l'exérèse chirurgicale des tissus éléphantiasiques, suivie d'une reconstruction scrotale ${ }^{1-10}$. Cette reconstruction scrotale est faite le plus souvent à partir des parties crânio-latérales et crânio-dorsale du scrotum qui sont le plus souvent préservées. Cette méthode, utilisée par de nombreux auteurs, ainsi que par notre équipe, semble donner un bon résultat fonctionnel et esthétique; les résultats de cette chirurgie sont excellents avec peu de récidives selon la littérature $1^{, 5,6,8-10}$. Nous n'avons pas observé de récidive après un recul de 4 ans. Quant à l'utilisation de greffes de peau libre mince, cette technique peut modifier la régulation thermique locale testiculaire et perturber la spermatogenèse ${ }^{1}$. Des auteurs tels 
que Prica et al. ${ }^{11}$ ont testé l'utilisation d'un lambeau myocutané du muscle gracile avec de bons résultats sur le plan esthétique et fonctionnel, mais sans avoir évalué la fertilité.

En ce qui concerne la greffe du pénis, comme le rapportent des auteurs ${ }^{1,5,6,8-10}$, il est préférable d'utiliser une peau libre mince qui doit être posée de façon spiralée afin d'éviter une rétraction longitudinale ou circulaire sur le pénis. La reconstruction du fourreau pénien était envisagée dans un deuxième temps opératoire chez notre patient. II ne s'est pas présenté et nous l'avons retrouvé près de 4 ans plus tard avec un excellent résultat esthétique et fonctionnel. L'ablation de la masse scrotale a probablement facilité le drainage lymphatique pénien, permettant la résorption de I'œdème ainsi que l'assouplissement et la revitalisation de la peau pénienne de notre patient.

L'éléphantiasis du scrotum et du pénis présente un formidable défi de reconstruction chirurgicale. Le bon résultat esthétique et fonctionnel obtenu ainsi que le faible taux de morbidité indiquent le choix de cette technique qui améliore la qualité de vie du patient.

\section{Conclusion}

L'éléphantiasis pénoscrotal est une affection rare, dont l'intérêt de l'étude réside dans sa prise en charge thérapeutique. II nécessite un bilan étiologique judicieux qui ne devrait pas empêcher ou retarder le traitement qui est toujours chirurgical. Les facteurs déterminant le choix de la technique opératoire sont : le type d'éléphantiasis (pénien, scrotal, péno-scrotal), la préservation ou non des parties crânio-latérales et crânio-dorsale du scrotum, l'étiologie (rétrécissement urétral, tumeur pelvienne, etc.), mais également les préférences du chirurgien.
Competing interests: None declared.

This paper has been peer-reviewed.

\section{Références}

1. Slama A, Jaidane M, Elleuch A, et al. Traitement chirurgical d'un éléphantiasis pénoscrotal. Prog Urol 2002:12:337-9

2. Dianzani $C$, Gaspardini $F$, Persichetti $P$, et al. Giant scrotal elephantiasis: an idiopathic case. Int $J$ Immunopathol Pharmacol 2010;23:369-72.

3. Aguiar-Santos AM, Leal-Cruz M, Netto MJ, et al. Lymph scrotum: an unusual urological presentation of lymphatic filariasis. A case series study. Rev Inst Med Trop Sao Paulo 2009;51:179-83.

4. Zugor V, Horch RE, Labanaris AP, et al. Penoscrotal elephantiasis: diagnostics and treatment options. Urologe A 2008;47:472-6. http://dx.doi.org/10.1007/s00120-007-1379-5

5. Halila M, Ounaes $A$, Saadani H, et al. L'éléphantiasis scrotal. Prog Urol 2003;13:140-2.

6. Ndoye A, Diao B, Fall PA, et al. Eléphantiasis des organes génitaux externes: aspects cliniques et thérapeutique. e-mémoires de l'Académie Nationale de Chirurgie 2003;2:39-42.

7. Masia DR, Castus P, Delia G, et al. A case of iatrogenic scrotal elephantiasis: reconstruction of the scrotal purse and the cutaneous sleeve of the penis with local skin flaps. Ann Chir Plast Esthet 2008;53:79-83. http://dx.doi.org/10.1016/i.anplas.2007.02.001

8. Franco Mora MC, Pichín Quesada A, Giraudy Simón G, et al. External genitalia lymphedema. Case report. Arch Esp. Urol 2007;60:688-92.

9. Garaffa G, Christopher N, Ralph DJ. The management of genital lymphoedema. BJU Int 2008;102:480-4. http://dx.doi.org/10.1111/i.1464-410X.2008.07559.x

10. Tazi MF, Ahallal Y, El Fassi MJ, et al. L'elephantiasis Pénoscrotal, diagnostic et prise en charge (A propos de 3 cas). J Maroc Urol 2009;16:17-20.

11. Prica S, Donati OF, Schaefer DJ, et al. Genital elephantiasis: reconstructive treatment of penoscrotal lymphoedema with a myocutaneous M. gracilis flap. Experiences from a District Hospital in Ethiopia. Handchir Mikrochir Plast Chir 2008;40:272-8. http://dx.doi.org/10.1055/s-2008-1038476

Correspondence: Dr. Angoran Dekou, Service d'urologie du CHU de Cocody, BPV 13 Abidjan, Côte d'Ivoire; dekouangoran@yahoo.fr 